\title{
REGIMES OF IGNITED OPERATION
}

L. Bromberg, D.R. Cohn and J. Fisher

March 1979

M.I.T. Plasma Fusion Center Report RR-79-3 
Regimes of Ignited Operation ${ }^{x}$

L. Bromberg, D. R. Cohn and J. Fisher ${ }^{\dagger}$

M.I.T. Plasma Fusion Center ${ }^{x x}$

and

Francis Bitter National Magnet Laboratory ${ }^{\dagger \dagger}$

M.I.T. Plasma Fusion Center Report RR-79-3

$x$ Supported by U.S. D.O.E. Contract EG-77-S-02-4183.A 002

† C.S.Draper Labotarory

xx Supported by U.S. Department of Energy

tt supported by N.S.F. 
Abstract

Regimes of ignited operation are explored in terms of a general four dimensional requirement on $n \tau_{e} n \tau_{i}, T_{i}$ and $T_{e}$. The conditions under which ignited operation occurs with $T_{e}>T_{i}$ and $T_{i}>T_{e}$ are found. The amount of electron-ion decoupling and the required value of $n$ r.e at ignition are determined as functions of the ion temperature and the ratio of the electron energy confinement time to the ion energy confinement time. Effects of anomalous transfer of alpha particle energy to the ions are considered. Thermal stability characteristics are determined in the context of the four dimensional ignition requirement. An empirical scaling law for the electron energy confinement time and the neoclassical transport description of the ion energy confinement time are used to project specific features of ignited operation in recent next step tokamak reactor designs. It is found that the ignition requirement on $n \tau_{e}$ can be significantly reduced by operating in the . $T_{i}>T_{e}$ regime at high ion temperatures. This reduction in $n \tau_{e}$ leads to a considerable reduction in the neutral beam energy required for adequate penetration in a full density startup scenario. The ion temperature dependence of characteristic thermal runaway times has also been determined for the next step reactor designs. The thermal runaway times are very short (on the order of $\tau_{e}$ ) until ion temperatures approaching $50 \mathrm{keV}$ are reached. 


\section{Introduction}

Ignition will play an important role in the operation of future major tokamak reactors'. The purpose of this paper is to determine the features of various regimes of ignited operation and to develop a more complete picture of the possible characteristics of these devices.

Ignition is defined as the thermal equilibrium condition in which the fusion power trapped in the plasma is sufficient to balance the plasma losses. The fusion reaction thus becomes self-sustaining. The very high values of $Q$ (Fusion power/Auxiliary Heating power) which are likely to be needed for a pure fusion tokamak reactor $(Q>20)$ are most readily obtained by operating at or very close to ignition. Ignited operation also obviates potential problems associated with steady state auxiliary heating.

The ignition condition is usually given as a two-dimensional requirement on the confinement quality parameter $n \tau_{E}$ and the plasma temperature, where $n$ is the plasma density and $\tau_{E}$ is the global energy confinement time that characterize the non-radiative energy losses. The electron and ion temperature are assumed equal. In this paper we determine the general conditions which lead to regimes where the electron temperature is greater or less than the ion temperature at ignition. The consequences of temperature decoupling on the general ignition requirement are then determined.

The general ignition condition is developed as a four dimensional requirement on $n \tau_{e} n \tau_{i}$ $T_{i}$ and $T_{e}$ in a plasma with no impurities and no cyclotron radiation losses. Here $\tau_{e}$ and $\tau_{i}$ are the electron and ion energy confinement times and $T_{\rho}$ and $T_{i}$ are their respective temperatures. Thermal stability properties of ignited plasma are examined in this context. Regimes of stable operation are then determined.

The special case where the ion energy confinement is considerably better than the electron energy confinement is studied. The investigation of this case is motivated by recent results in the PLT tokamak; in these experiments adverse effects of the trapped ion mode have not appeared at very low values of collisionality and the ion energy transport seems to be accounted for by 
neoclassical theory. ${ }^{2}$ Another case which is of interest is the case where the anisotropic velocity distribution of alpha particles leads to anomalously fast transfer of alpha power to the ions. ${ }^{3}$ Finally, an empirical scaling law for the electron energy confinement time is used as a basis for projecting features of ignited operation in recent tokamak reactor designs; heating and toroidal beta requirements are determined.

\section{Ignition Regimes: Equilibrium}

The plasma power balance equations are given by

$$
\frac{i}{2} \frac{d n T_{e}}{d t}=n^{2} R_{\alpha} F_{e}+\frac{3}{5} \frac{n\left(T_{l}-T_{e}\right)}{T_{i e}}-\frac{n T_{e}}{2 \tau_{e}}-n^{2} R_{b}
$$

for the electrons and

$$
\frac{1}{2} \frac{d n T_{i}}{d t}=n^{2} R_{\alpha}\left(1-F_{e}\right)-\frac{3}{5} \frac{n\left(T_{i}-T_{e}\right)}{\tau_{i \ell}}-\frac{n T_{i}}{2 \tau_{i}}
$$

for the ions. Parabolic profiles are assumed in these equations; plasma density and temperatures are represented by their values on axis. It is assumed that no impurities are present and cyclotron radiation and ohmic heating are ignored. These additional effects are included in a later section. The subscripts i,e refer to the ions and electrons respectively. $\tau_{i}$ and $\tau_{e}$ refer to the ion and electron energy confinement times. $\tau_{i e}$ is the electron-ion equilibration time. $R_{\alpha}$ is defined by

$$
F_{e} R_{\alpha}=\frac{1}{4 n^{2}} \overline{n(r)^{2}\langle\sigma v\rangle F_{e}} E_{\alpha}
$$

where $E_{\alpha}$ is the initial energy of the alpha particles. $R_{b} n^{2}$ is the area averaged Bremstrahlung losses.

$F_{e}$ is the fraction of the alpha energy power given to the electrons. Classically $F_{e}$ dependes only on the electron temperature in the range of interest. However, it has been pointed out by Molvig that the alpha particles may have a non-classical slowing down time. ${ }^{3}$ In this case, it is predicted that most of the energy goes directly into the ions.

Ignition is defined as the condition that results in

$$
n \frac{d T_{e}}{d t}=n \frac{d T_{i}}{d t}=0
$$


Under these circumstances, the alpha particle power balances the plasma losses, and the plasma is energetically self-sustaining.

The ignition condition is obtained by adding (1) and (2) at ignition:

$$
\left\langle n \tau_{e}\right)_{i g n}=\frac{T_{e}}{R_{a}-R_{b}}\left(1+\frac{T_{i} \tau_{e}}{T_{e} T_{i}}\right)
$$

If $T_{i}=T_{e}$ the usual ignition condition is obtained. If a global energy confinement time $\tau_{E}$ is defined as

$$
\tau_{E}=\frac{\tau_{i} \tau_{e}}{\tau_{e}+\tau_{i}}
$$

then it can be seen from (4) that the confinement quality parameter $\left(n \tau_{E}\right)_{i g n}$ depends only on the plasma temperature.

From (1) and (2) it is possible to find the direction of power flow between the ions and the electrons. By simple algebraic manipulation, it is found that when Bremstrahlung losses (which are small in the region of interest) are neglected

$$
\left(T_{i}-T_{e}\right)=\frac{5\left(T_{e} \tau_{i e}\right)}{3 \tau_{e}}\left(1-F_{e}-F_{e} \frac{T_{i}}{T_{e}} \frac{T_{e}}{T_{i}}\right)
$$

As $\tau_{i e} \sim T_{e}^{3 / 2} / n,(5)$ can be rewritten as

$$
\left(T_{i}-T_{e}\right) \sim \frac{T_{e}^{5 / 2}}{n \tau_{e}}\left(1-F_{e}-F_{e} \frac{T_{i}}{T_{e}} \frac{T_{e}}{T_{i}}\right)
$$

It can be seen from (6) that the direction of the power flow depends only on the sign of

$$
1-F_{e}-F_{e} \frac{\tau_{e}}{\tau_{i}}
$$

It can also be seen that the electron-ion temperature decoupling increases with increasing electron temperature and with decreasing $\left(n \tau_{\rho}\right)$.

Figure I shows the ratio $\tau_{d} l \tau_{i}$ for which $T_{l}=T_{e}$ as a function of the electron temperature assuming that the alpha particles slow down classically. As the ratio of electron to ion energy

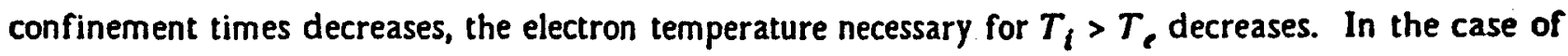
complete alpha energy transfer to the ions $\left(F_{e}=0\right)$ due to instabilities the ion temperature is always 
greater than or equal to the electron temperature.

Combining (4) and (5) one can determine the electron temperature at ignition $T_{e, ~}$ ign and $\left(n \tau_{e}\right)_{i g n}$ as function of $T_{i}$ for fixed $\tau_{d} \tau_{i}$. Figure 2a shows $T_{e, i g n}$ as a function of $T_{i}$ assuming $\tau d \tau_{i}=1$. Two cases are shown: one is for classical slowing down of the alpha particles, giving energy to both ions and electrons. The other case is when all the alpha particle energy is released to the ions, as a result of an alpha driven instability. Figure $2 b$ shows the values of $\left(n \dot{\tau}_{e}\right\rangle_{i g n}$ as $a$ function of the ion temperature.

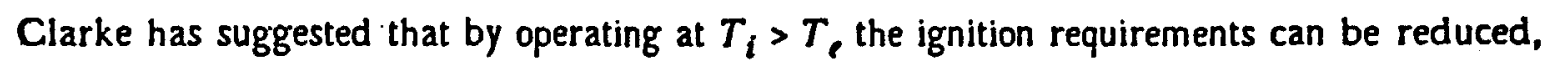
facilitating operation at high ion temperatures where thermal stability is more likely to occur. ${ }^{4}$ Figures $3 \mathrm{a}$ and $3 \mathrm{~b}$ show how the amount of ion-electron decoupling and the reduction in $\boldsymbol{n}_{\boldsymbol{e}}$ depend upon the ratio of $\tau_{d} d \tau_{i}$. Significant reductions in $n \tau_{p}$ can be obtained by operation at low values of $\tau_{d} d \tau_{i}$ which facilitate ion-electron decoupling. Classical alpha slowing down has been assumed in Figures $3 \mathrm{a}$ and $3 \mathrm{~b}$.

\section{Ignition Regimes: Stability}

It is possible to determine plasma stability properties using the simplified model described in the last section. The stability properties can be shown to depend only on $T_{e}$ and $T_{i}$ and the ignition condition (3).

It is assumed that $\left(n \tau_{e}\right)$ is independent of the electron and ion temperatures; the empirical scaling of the electron energy confinement time seems to indicate only a weak temperature depedence." It is also assumed that $\left(n \tau_{l}\right)$ does not depend on the electron temperature.

The procedure to examine the stability is as follows: a temperature excursion on the ions is specified. The electron temperature perturbation which satisfies

$$
\frac{d n T_{e}}{d t}=0
$$

is then determined. The only time dependence which remains is that of the ion temperature. If the perturbation increases the system is unstable. 
The time depedent system is then

$$
\frac{1}{2 n} \frac{d T_{i}}{d t}=R_{\alpha}\left(1-F_{i}\left(T_{l}\right)\right)-\frac{3\left(T_{i}-T_{l}\right)}{5 n \tau_{i e}}-\frac{T_{i}}{2 n \tau_{i}}
$$

where $T_{e}$ at $t=0$ is determined from

$$
0=R_{a} F_{e}+\frac{3\left(T_{i}-T_{e}\right)}{5 n \tau_{i e}}-\frac{T_{e}}{2 n \tau_{e}}-R_{b}\left(T_{e}\right)
$$

Cyclotron emission has been neglected in (8). The effect of cyclotron emission is model dependent, and will be included in the next section.

The ion confinement quality parameter is assumed to scale with ion temperature as $\left(n \tau_{i}\right) \sim T_{i}^{\alpha}$. Then the required value of a that results in a neutrally stable system is found by taking the derivative of $(7)$ with respect to $T_{i}$ and setting

$$
\frac{d}{d T_{i}} \frac{1}{2 n} \frac{d T_{i}}{d t}=0
$$

In order to facilitate the calculation, finite differences rather than derivatives are used. After some algebraic manipulation, it is found that the value of a that results in a neutrally stable system is given by

$$
a_{\text {neut stab }}=\frac{1}{\ln \left(\tilde{T}_{i} / T_{i}\right)} \ln \left(0.5 \frac{\tilde{T}_{i}}{n \tau_{i 0}} \frac{1}{R_{\alpha}\left(\tilde{T}_{i}\right)-\frac{\tilde{T}_{e}}{2 n \tau_{e}}-R_{b}\left(\tilde{T}_{e}\right)}\right)
$$

where $\tilde{T}_{i}=T_{i}+\delta T_{i}$ and $\left(n \tau_{i n}\right)$ is the value of $\left(n \tau_{i}\right)_{i g n}$ evaluated at $T_{i} \cdot \tilde{T}_{e}=T_{e}+\delta T_{e}$ is calculated from (8). The term neutral stability refers to the conditions under which a perturbation $\therefore$ on an equilibrium does not grow or damp away.

Figure 4 shows the value of $a_{\text {neut stab }}$ as a function of the ion and electron temperatures for classical slowing down of the alpha particles. As the ion temperature increases, the tolerable value of $\alpha_{\text {neut stab }}$ increases because the system is more stable. Note that for fixed ion temperature the allowed values of $\alpha$ for stability increase with increasing electron temperatures (that is, the system is more stable). The case with anomalous slowing down results in slightly less stable plasmas than the classical case. For neoclassical scaling with $\alpha=0.5$, a value of $T_{i} \sim 50 \mathrm{keV}$ is needed for thermally 
stable operation.

The stability of an ignited plasma satisfying the neoclassical energy confinement for the ions is considered. This corresponds to setting $\alpha=0.5$ in the above analysis. As before, it is assumed that the electron energy confinement time is independent on temperature, and that the density is constant during a perturbation. The time that it takes the plasma to run away can be defined by

$$
\tau_{\text {run away }}=P\left(T_{i}\right) \frac{d t}{d P\left(T_{i}\right)}
$$

where $P\left(T_{i}\right)$ is the total power balance for the plasma. For the temperature perturbation described above,

$$
P\left(T_{i}\right)=R_{\alpha}\left(T_{i}\right)\left(1-F_{e}\right)-\frac{T_{i}}{2 n \tau_{i}}-\frac{3\left(T_{i}-T_{e}\right)}{5 n \tau_{i \ell}} .
$$

After some manipulations on (7), it is found that

$$
\frac{\tau_{\text {nunaway }}}{\tau_{e}}=\frac{1}{2 n \tau_{e}}\left(\frac{d P\left(T_{i}\right)}{d T_{i}}\right)^{-1}
$$

Figure 5 shows the ratio $\tau_{\text {runaway }} / \tau_{e}$ as a function of $T_{e}$ and $T_{l}$. As expected, going to higher ion temperatures improves the stability of the system. However, as the electron temperature increases at fixed ion temperature, the ratio $\tau_{\text {nunaway }} / \tau$, decreases. This decrease is due to the fact that as the electron temperature increases the coupling between the electrons and the ions decreases. In addition, as the electron temperature increases, a larger fraction of the alpha power goes directly into the ions. Both of these effects tend to speed up the ion response.

In order to check the validity of these simple calculations, the system (1) and (2) has been augmented with ion density and alpha energy density equations and solved without the assumption that the electron temperature satisfies (8). In this manner density changes and finite alpha slowing down time are included in the model. The stability properties are then derived by solving for the eigenvalues of the system which has been linearized around the equilibrium point. ${ }^{5}$ The general conditions for stability are not changed significantly from those derived using the simplified system, as long as the alpha slowing down time remains short compared to the $T_{e} T_{i}$ dynamics (usually satisfied in the regimes of interest), and the density does not change significantly in the times of 
interest for the thermal stability. This last condition is satisfied when either $(i)$ there is a feeback control maintaining constant density, or (ii) the particle confinement time is long compared with the times of interest and is relatively independent of temperature. It should be noted that as the thermal runaway time becomes comparable to the particle confinement time, then a specific model for the time behavior of density becomes important. ${ }^{6}$ Considerations of this effect are outside the scope of this paper.

\section{Ignition Regimes of Recent Next Step Tokamak Reactor Designs}

The features of ignited operation in two specific reactor designs are considered. These reactor designs are a copper magnet high field ignition test reactor (ITR), and a next step reactor design which uses a superconducting toroidal field magnet (TNS)!.

The study of ignition regimes in specific tokamak devices depends upon the particular description which are used for the electron and ion energy confinement times. In order to make an assessment we assume that the electron energy confinement time is described by the empirical scaling relation $^{7}$

$$
\tau_{e}=\frac{1}{2} \tau_{e m p}=1.910^{-19} n a^{2}
$$

where $n$ is the central density is $\mathrm{cm}^{-3}$ and $a$ is the minor radius in $\mathrm{cm}$. The factor of 0.5 in (12) is due to the fact that in reference 7 the empirical confinement time is defined as the global energy confinement time; here. $\tau$, refers only to the electron loss channel. It is assumed that elongation does not improve the electron energy confinement time over the value given by (12). It is also assumed that the ion energy confinement is described by neoclassical theory. The cyclotron emission has been included by assuming that ${ }^{-8}$

$$
P_{c y c}=2.410^{-14} B_{T}^{5 / 2} n^{1 / 2} a^{-1 / 2} T_{e}^{11 / 4}\left(1+\frac{T_{e}}{204}\right)
$$

where a reflectivity of $90 \%$ has been assumed.

The general parameters of the illustrative ITR and TNS designs are shown in Table $\mathbf{I}$.

Because the energy confinement scaling with plasma and geometrical parameters has been 
assumed, the ignition criteria can now be defined in terms of

$$
n_{i g n}=n_{i g n}\left(T_{i}\right)
$$

The degree of electron-ion decoupling and the value of $\left(n \tau_{\rho}\right)_{i g n}$ depend on the ratio between the electron and the ion energy confinement times, $\tau_{d} d \tau_{l}$. Assuming empirical scaling for the electrons and neoclassical for the ions, then

$$
\frac{\tau_{e}}{\tau_{i}} \sim \frac{n^{2} A^{3 / 2} q^{2}}{T_{i}^{1 / 2} B_{T}^{2}} \sim \frac{\beta_{T}^{2} A^{3 / 2} q^{2} B_{T}^{2}}{T_{i}^{1 / 2}\left(T_{i}+T_{e}\right)^{2}}
$$

Figure 6 shows $\tau d \tau_{i}$ at ignition as a function of the ion temperature for both the ITR and the TNS. As the ITR has a built-in compression, what is shown are the minimum and maximum values of $\tau_{d} \tau_{i}$ at a given ion temperature (corresponding to the compressed and uncompressed states, respectively). The ITR compressed plasma and the TNS are characterized by similar values. of $\tau d \tau_{i}$

In Figure $7 \mathrm{a}$ the values of the average toroidal beta required for ignition, $\beta_{T}$ ign are plotted for the ITR and the TNS as a function of the ion temperature. Classical transfer of alpha energy is assumed. Note that $\beta_{T}$ ign increases with increasing ion temperatures for $T_{i}>15-20 \mathrm{keV}$. Figure $7 \mathrm{~b}$ shows the values of $\beta_{T}$ ign for both the ITR and the TNS obtained by arbitrarily assuming that the ion and the electron temperatures are equal and assuming that the total energy confinement time is given by the empirical scaling law, $\tau_{E}=\tau_{e m p}$. It can be seen that the ion-electron decoupling shifts the minimum beta to higher temperatures. This shift occurs both because of the decrease in

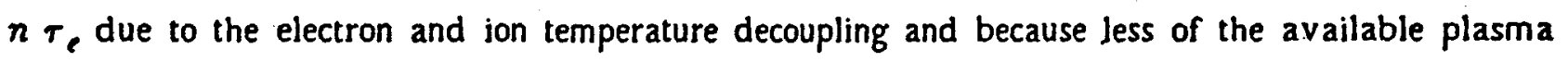
pressure is allocated to the electrons. Nonclassical slowing down of the alpha particles increases this. shift and leads to a minimum in $B_{T \text { ign }}$ at $T_{l} \sim 25 \mathrm{keV}$. Figures $7 \mathrm{a}$ and $7 \mathrm{~b}$ also show that in order to operate at a neutrally stable point for neoclassical scaling, which occurs when $T_{i}>50 \mathrm{keV}$ (see Figure 4), the required value of $\beta_{T}$ must be $\sim 1.5$ times greater than the minimum value which is obtained at $\sim 15 \mathrm{keV}$. Thus, if the empirical scaling relation given by (12) and neoclassical description of the ion confinement are applicable, a significant price in beta must be paid to achieve 
naturally stable plasmas. It may be, however, that an increase in trasport due to the onset of balloning modes may lead to self regulation. ${ }^{9}$

The runaway time, as defined in section III, is shown both for ITR and the TNS in Figure 8. The runaway time for the ITR is only weakly dependent on whether the plasma is in the compressed or uncompressed state. As the temperature increases, the system becomes more stable. However, thermal stability does not occur until $T_{i} \sim 50 \mathrm{keV}$, in agreement with results from the previous. section (which did not include cyclotron emission). Not shown are the results for the case where the alpha particles slow down non-classically on the ions. Non-classical slowing down of the alpha particles has only a small effect on the thermal runaway time at a fixed ion temperature.

The auxiliary heating power required for heating the TNS to ignition at constant density has been calculated. It does not vary significantly with the ion temperature at ignition. Figure 9 shows the beam energy required for slightly peaked beam deposition profiles for both classical and anomalous alpha particle slowing down. The beam energy, already reduced by operating at high ion temperatures $^{10}$ in the case where $T_{p}=T_{l}$, is further decreased by ion-electron decoupling. The shifting of the magnetic axis due to finite beta effects has not been included in Figure 9.

\section{Summary}

The following aspects of ignited operation have emerged from the above considerations:

In the absence of impurities and cyclotron radiation, the ignition requirement is in general a four dimensional requirement on $\left(n \tau_{e}\right),\left(n \tau_{i}\right), T_{c}$ and $T_{i}$. Regions where $T_{e}>T_{i}, T_{i}=T_{e}$ and $T_{i}>T_{e}$ are determined by the ratio of the electron energy confinement time. to the ion confinement time and by the electron temperature. A low value of this ratio leads to the onset of the $T_{l}>T_{e}$ ignition regime at relatively low values of the electron temperature. This regime can also be made more accessible by an anomalous transfer of the alpha particle energy to the ions. Operation in the regime where $T_{i}$ is significantly larger than $T_{e}$ results in a significant reduction in $\left(n \tau_{e}\right)$ over the value that is determined by arbitrarily 
setting $T_{e}=T_{i}$.

Thermal stability must be viewed in the context of the four dimensional ignition requirement. The required dependence of the ion energy confinement time on ion temperature for neutral stability is calculated under the assumption of a weak temperature dependence of the electron energy confinement time. It is found that for the $\tau_{i} \sim T_{i}^{1 / 2}$ dependence of neoclassical scaling, stable operation leads to the requirement of a thermal equilibrium point with an ion temperature around $50 \mathrm{keV}$. The thermal runaway time increases very rapidly as this equilibrium point is approached.

If the ignition point is passed at low value of $T_{i}$ (i.e.; in the 5-10 keV range) due to initial overheating or inadequacy of thermal stability control, equilibrium may not be reestablished until $T_{i}$ is much greater than $50 \mathrm{keV}$. Thus a very large. change in temperature might occur without good control over the heating rate and profile. This uncontrolled temperature change could lead to pressure driven instabilities. These instabilities might result in a major disruption or in a rapid quenching of the burn by a sudden change in the temperature profile.

Continuous operation at an unstable four dimensional ignition point imposes requirements on both the accuracy of heating to the desired electron and ion temperatures and on the degree of fast response active thermal stability control which is required. If the heating source is turned off after producing a value of $\left(n \tau_{e}\right),\left(n \tau_{i}\right), T_{e}$ and $T_{l}$ which is not sufficiently close to a thermal equilibrium point, the active thermal stability control will not be effective.' (The case of very high $Q$ driven operation is similar to the case of fully ignited operation with the exception that a small amount of direct heating is used for thermal stability control; it is still necessary to run at relatively precisely determined values of $\left(n \tau_{e}\right) .\left(n \tau_{l}\right)$. $T_{p}$ and $T_{i}$ ). Because of the above considerations, it may be necessary to independently control the electron and the ion temperatures. Operation at a 
temperature where $T_{e}=T_{l}$ may make it easier to hit the thermal equilibrium point with sufficient accuracy. Thermal equilibrium control, such as the use of a high-Z noble gas impurity (e.g., xenon) to preset the ignition point may be desirable. In this way the thermal output power in a reactor can be increased while maintaining thermal equilibrium.

Direct heating to a thermal equilibrium point which tends towards thermal stability could eliminate the requirement for the fast time scale control. However, longer time scale control would still be necessary to deal with density changes and impurity buildup. In addition, the high temperatures which are required for thermal stability are likely to lead to an increase in the beta requirements.

Neoclassical scaling for the ion energy confinement time and an empirical scaling law for the electron energy confinement time are used as a basis for . projecting features of ignited operation for recent copper magnet ignition test reactor and superconducting magnet TNS designs. These features are very similar since the ratios of the electron to the ion energy confinement times are nearly the same for both designs. For classical alpha slowing down, the $\left(n \tau_{e}\right)$ required for ignition at $T_{i}=30 \mathrm{keV}$ is reduced by a factor of $\sim 2$ over that required if $T_{e}$ is arbitrarily set equal to $T_{i}$. This reduction in $\left(n \tau_{e}\right)$ would lead to a $40 \%$ reduction in neutral beam energy required for penetration. This reduction could be further increased by the presence of anomalous alpha slowing down. Determining $T_{e}$ and $T_{i}$ self-consistently also leads to an increase in the temperature at which the minimum beta required for ignition occurs.

In summary, there are a large number of regimes of ignited operation with varying features and requirements. The determination of the most desirable regime for future power reactor operation is contingent upon the acquisition of experimental information about the interplay between high temperature transport, beta limits, impurity effects, alpha particle induced instabilities and thermal stability control. 


\section{Acknowledgements}

The authors wish to thank Dr. J.F. Clarke for suggesting hot ion operation as a means to reduce $n \tau_{e}$ required for ignition and to facilitate the attainment of high temperature, thermally stable plasma. Discussions with Dr. K. Molvig concerning alpha particles instabilities are gratefully acknowledged. The authors are also indebted to Dr. K. Lackner for very useful discussions.

This work was carried out as part of a joint ignition experiment design with the Max-Planck Institute fur Plasmaphysik, Garching and the Divisione Fusione of CNEN, Frascati. 


\section{References}

1 For example, BROMBERG, L., COHN, D.R., WILLIAMS, J.E.C., M.I.T. Plasma Fusion Center Report RR-78-12 (Nov. 1978); Compact Ignition Experiment Internal Status Report, prepared by Max Plank Institut fur Plasmaphysik, Garching and the Divisone Fusione of CNEN, Frascati (1978); D. Steiner et al, ORNL TNS Study, ORNL/TNS Study, ORNL/TM 6720 (Nov 1978)

2 EUBANK, H., Bull Am Phys Soc 23745 (1978)

3 MOLVIG, K., private communication (Ignition Experiment Design Meeting, Cambridge, MA (Jan 1979))

4 CLARKE, J.F., private communication (Ignition Experiment Design Meeting, Cambridge, Ma (Jan 1979))

5 See, for example: YAMATO, H., OHTA, M., and MORI, S., Nucl Fusion 12604 (1972); EDELBAUM, T.N., et al., C.S. Draper Lab. Fusion Reactor Control Study, Second Annual Report, EPRI Research Project 546-2 (1978)

6 Houlberg, W.A., and CONN, R.W., Nucl Fusion 1981 (1979)

7 COHN, D.R., PARKER, R.R., and JASSBY, D.L. Nucl Fusion 1631 (1976); Jassby, D.L., COHN, D.R., and PARKER, R.R., Nucl Fusion 161045 (1976)

8 ATTENBERG, S.E., MARCUS, F.B., and MCALEES, D.G., ORNL/TM-5509 (Nov 1976)

9 OKABAYASHI, M., private communication

10 COHN, D.R., JASSBY, D.L., and KREISCHER, K., Nucl Fusion 181255 (1978) 
TABLE I

Plasma Major Radius, $R$ (m)

TNS

ITR *

Plasma Elongation

5.0

1.3

1.6

1.0

Compression Ratio

1.0

1.5

Plasma Minor Radius, a (m)

1.2

0.5

Plasma Current, $I_{p}$ (MA)

5.0

3.6

Field on Axis, $B_{T}(T)$

5.3

9.5

Steady State Burn Time ( $s$ )

500

10

Neutral Beam Power (MW)

50

15

Toroidal Field Coils

Supercond. Normal

Shielding of TF coils

SS balls None and borated water

* Compressed Plasma Parameters 


\section{FIGURE CAPTIONS}

Figure 1 Value of $\tau_{e} / \tau_{i}$ that results in $T_{e}=T_{j}$ as a function of $T_{e}$.

Figure 2 Value of (a) $\mathrm{T}_{\mathrm{e}}$ ign and $(\mathrm{b})\left(\mathrm{n}_{\mathrm{\tau}} \mathrm{e}\right)$ ign as a function of peak ion temperature, $T_{i}$. C is for classical slowing down of the alpha particles, and $A$ for anomalous slowing down on the ions $\left(F_{e}=0\right)$. $\tau^{\prime} e^{/ \tau}=1$.

Figure 3 Same as Figure 2, for $\tau_{e} / \tau_{i}=0,1$ and 2. Classical slowing down of the alpha particles is assumed in all cases.

Figure 4 Contours of constant value of $\alpha_{\text {neut stab }}$ that result in a neutrally stable system as a function of peak electron and ion temperatures. For values of $a<\alpha_{\text {neut stab }}$ the system is stable. Classical alpha slowing down is assumed.

Figure 5. Contours of constant ratios of $\tau_{\text {runaway }} / \tau_{E}$ as a function of peak electron and ion temperatures. Classical alpha slowing down is assumed.

Figure 6 Ratio of electron energy confinement time to ion energy confinement time at ignition for the TNS and the ITR. The curves for the ITR refer to the uncompressed and compressed states.

Figure 7 Peak ion temperature dependence of toroidal beta at ignition for the ITR and TNS designs: (a) self-consistent calculations (b) arbitrarily assuming that $T_{e}=T_{i}$. Curves are shown for both compressed and uncompressed ITR plasmas. Classical slowing down of the alpha particles is assumed.

Figure $8 \tau_{\text {runaway }}$ as a function of peak ion temperature. $\tau_{\text {runaway }}$ is only weakly dependent on whether the alpha particles slow down classically or anomalously. Curves are shown for the TNS design and the ITR design with a compressed plasma. 
Figure 9 Peak ion temperature dependence of neutral beam energy requirement for the TNS design. A full density start-up with slightly peaked beam deposition profiles is assumed. Curves are shown for both classical (C) and anomalous (A) slowing down of the alpha particles. 


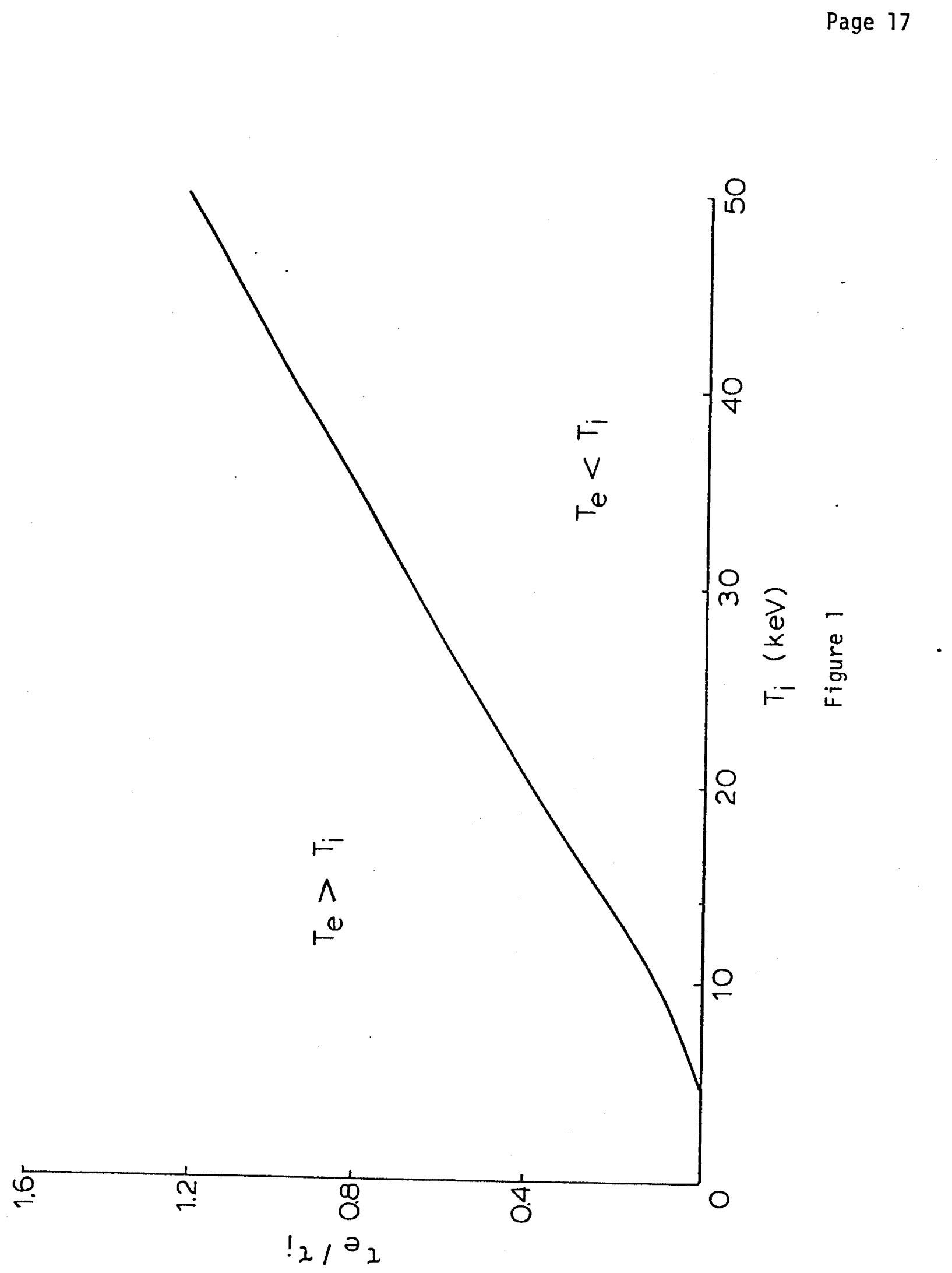




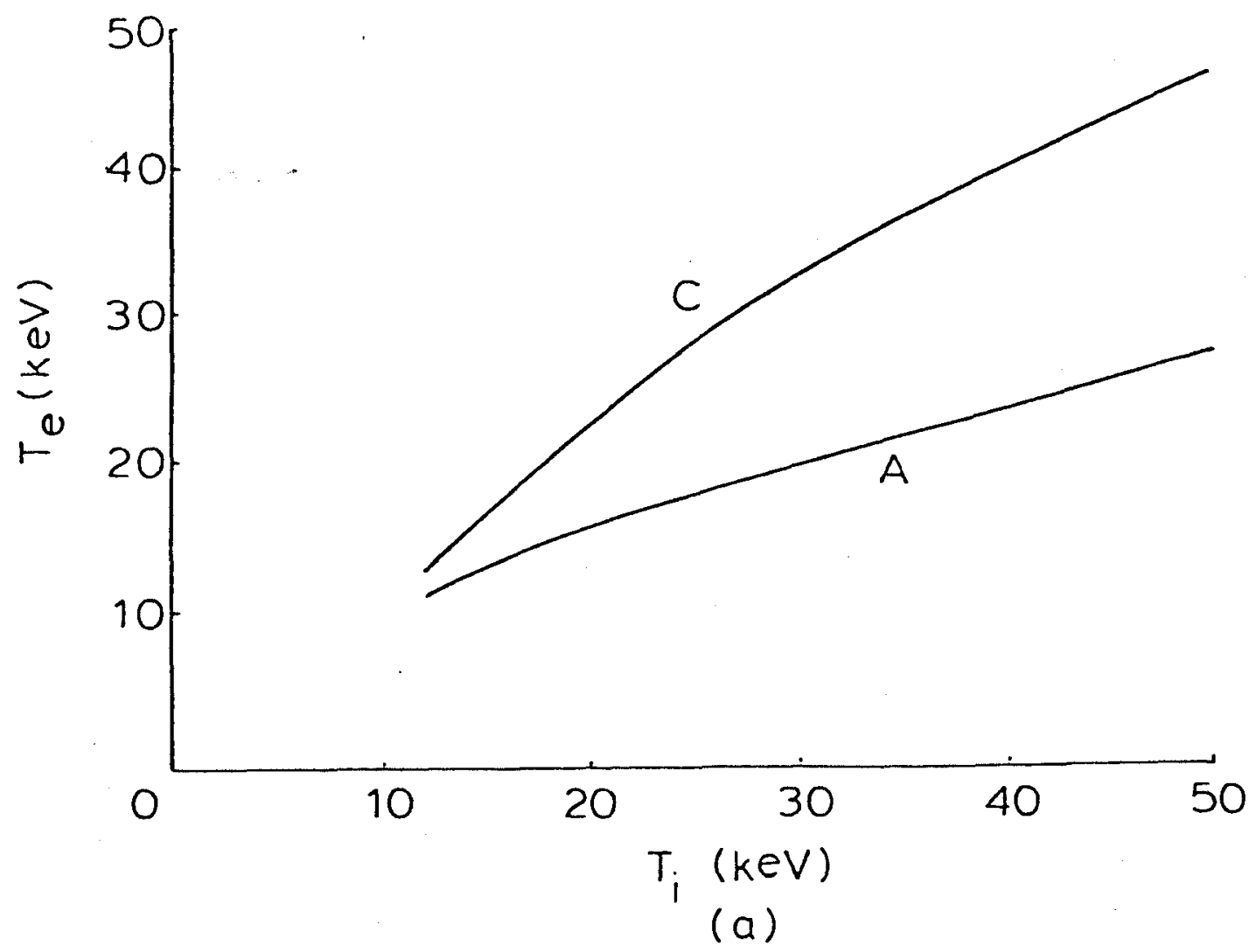

Page 18

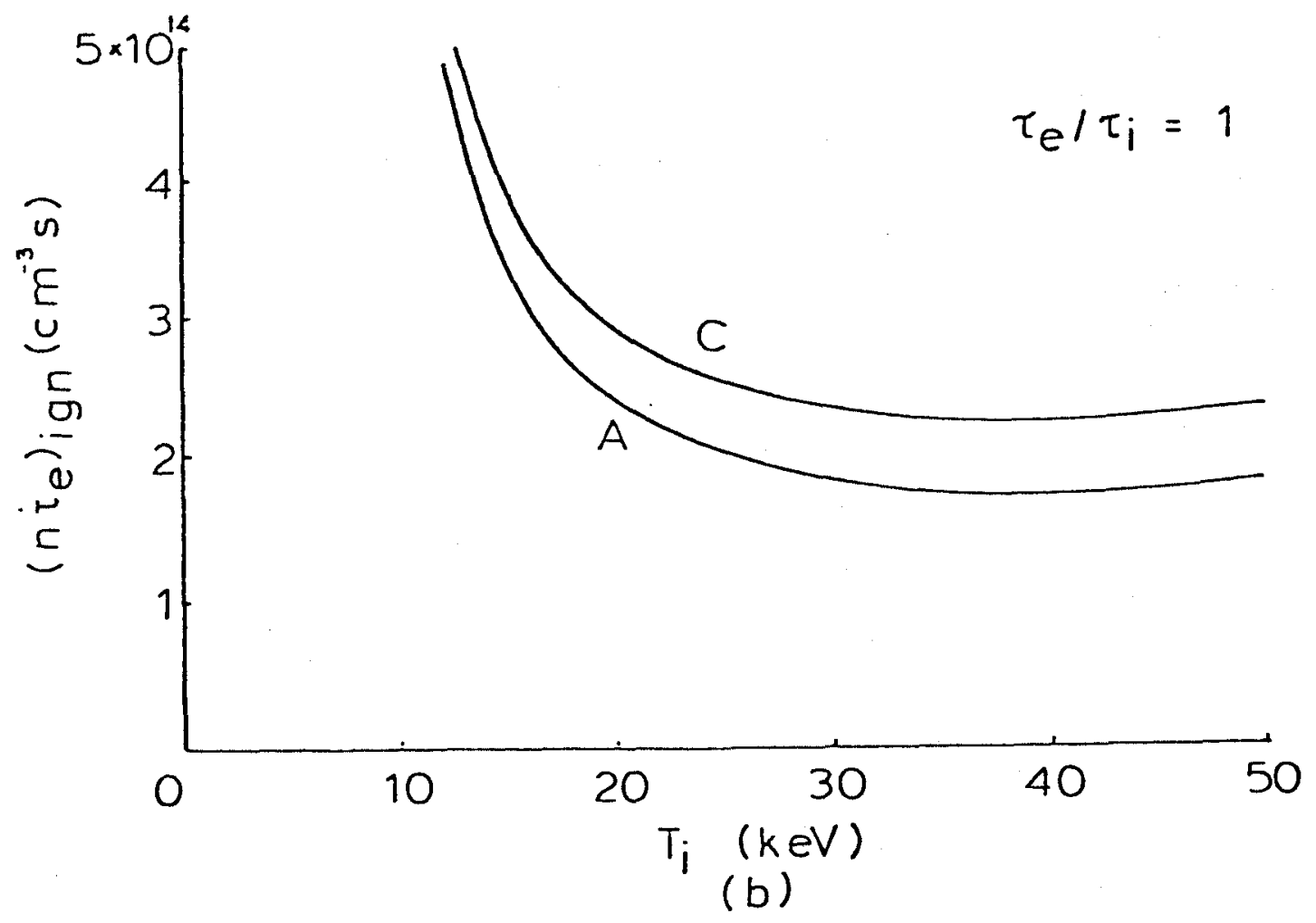

Figure 2 

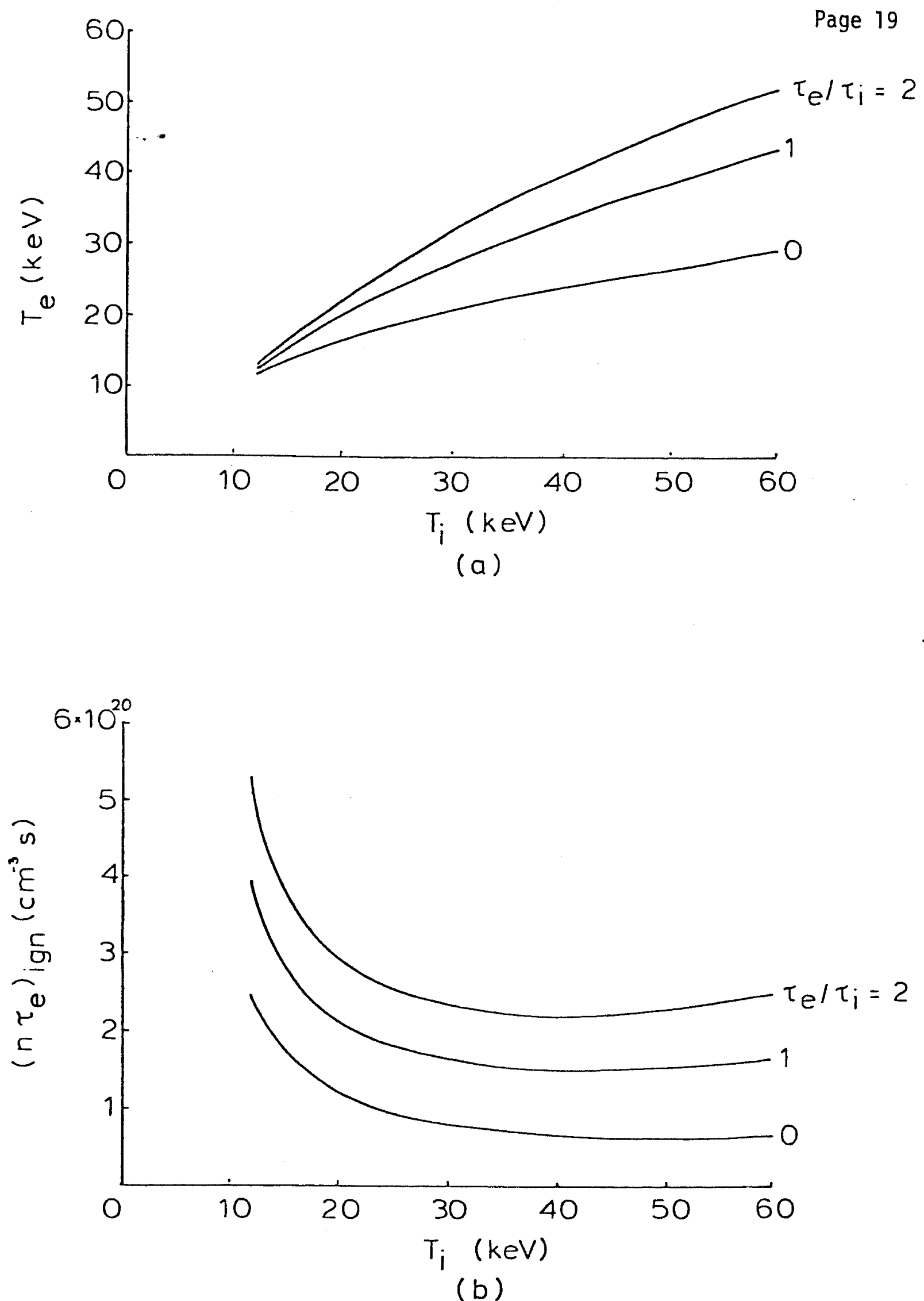

Figure 3 
Page 20

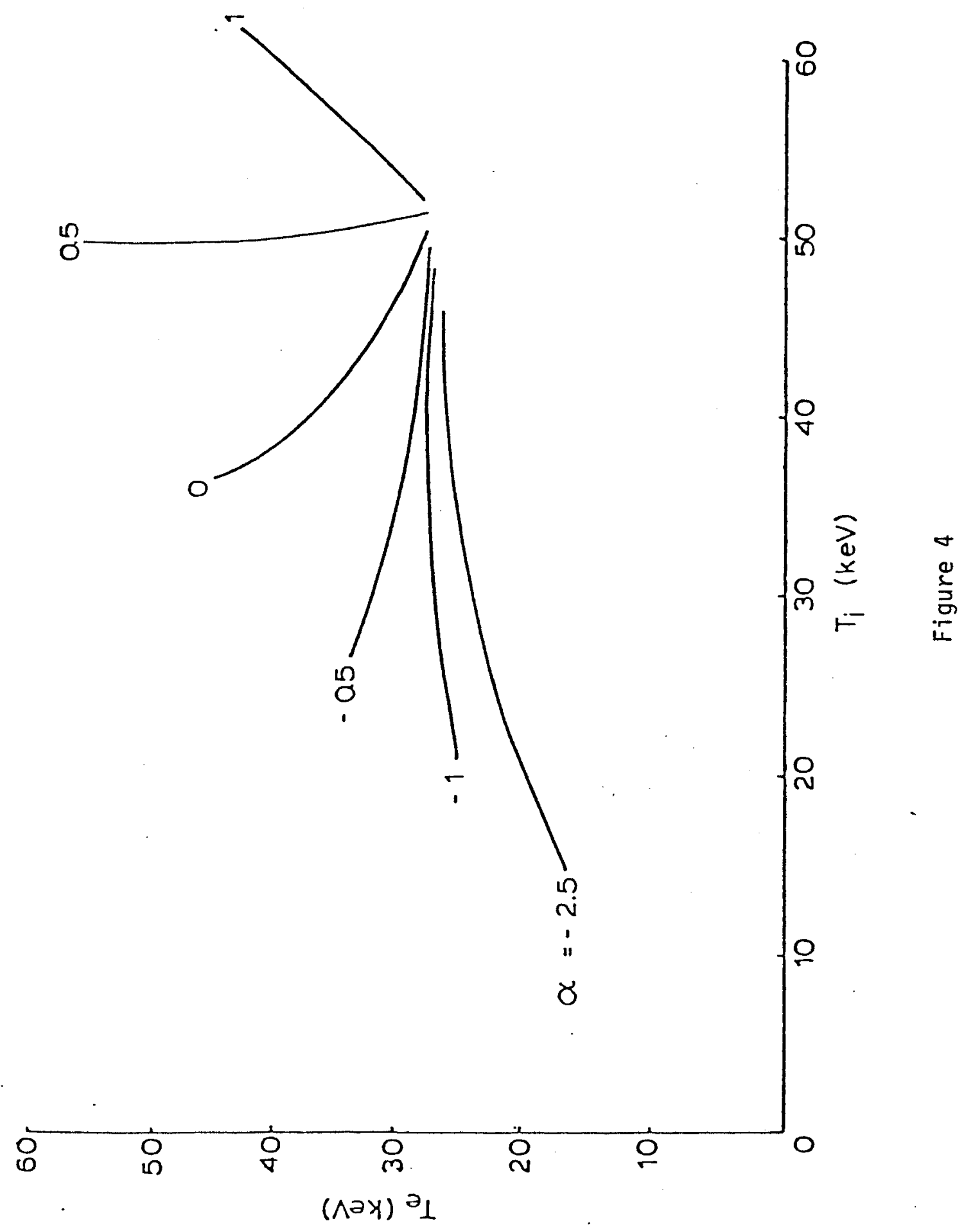


Page 21

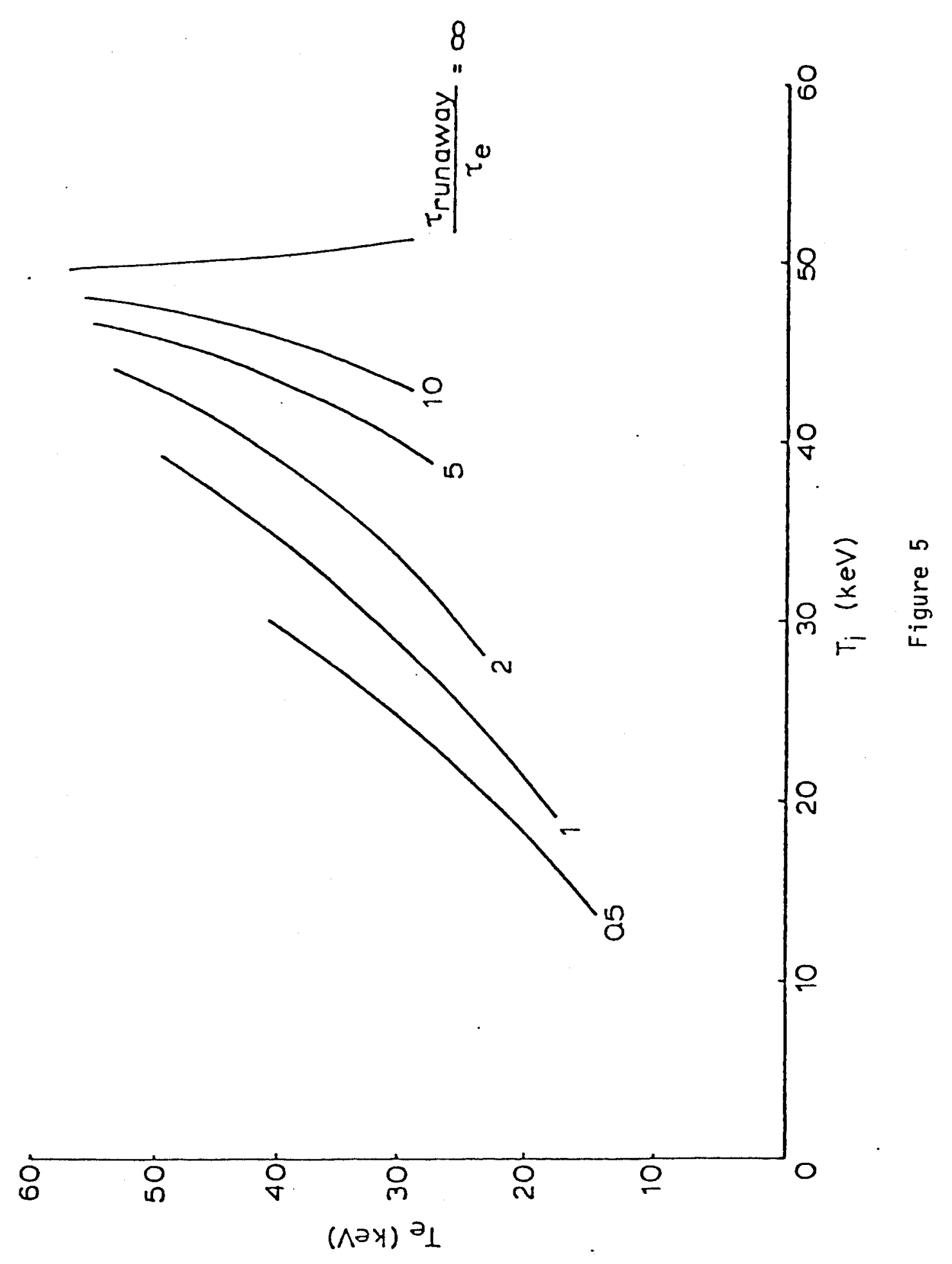


Page 22

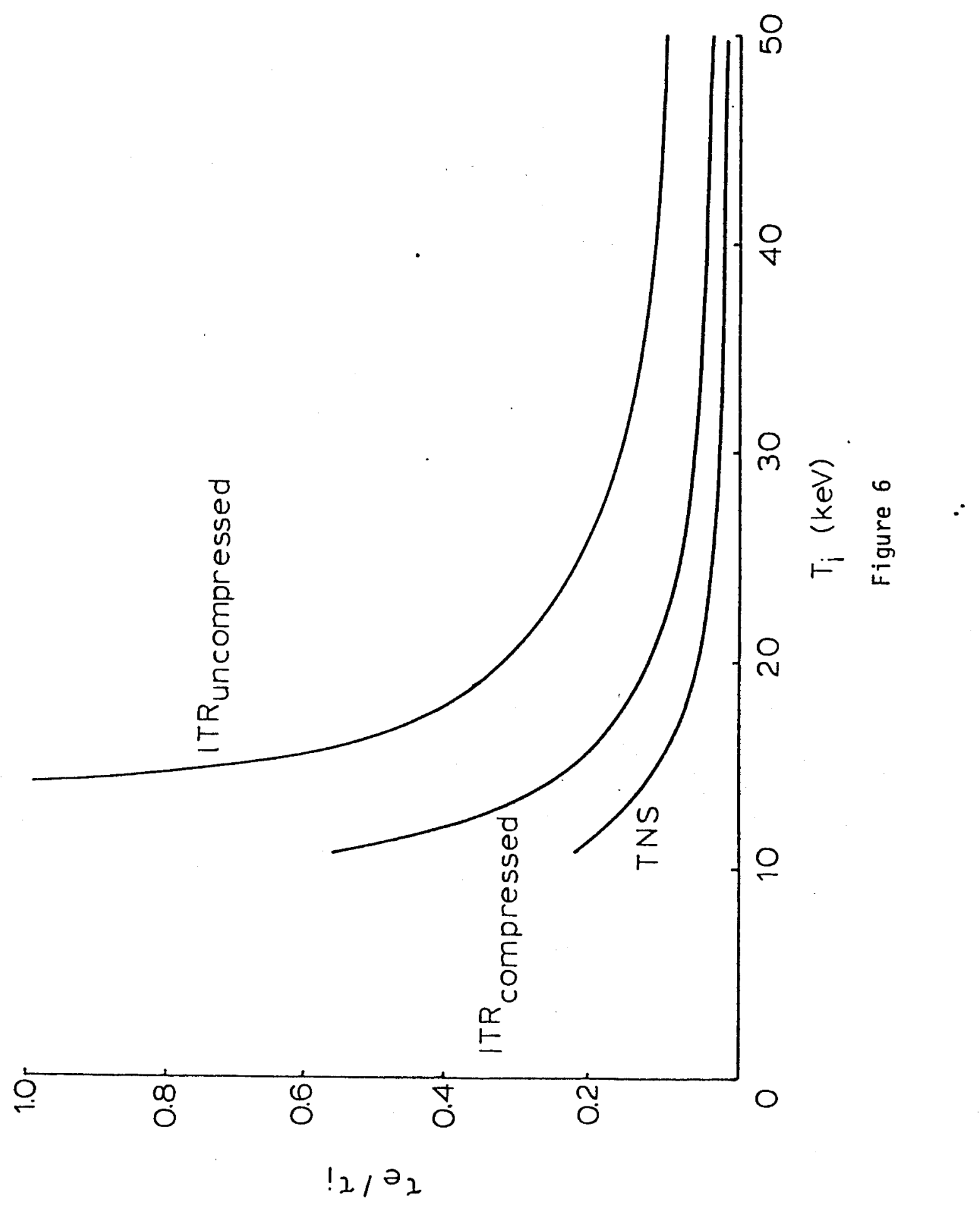


Page 23

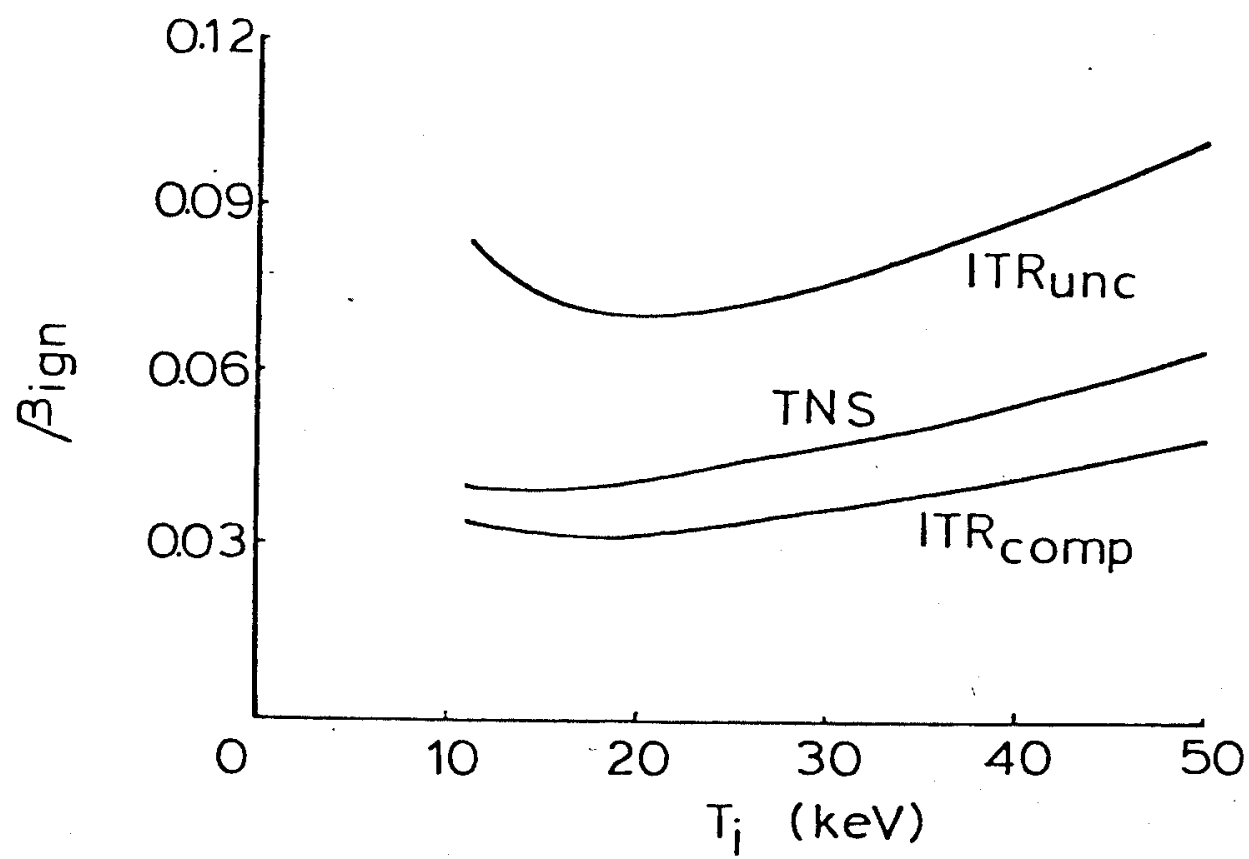

(a)

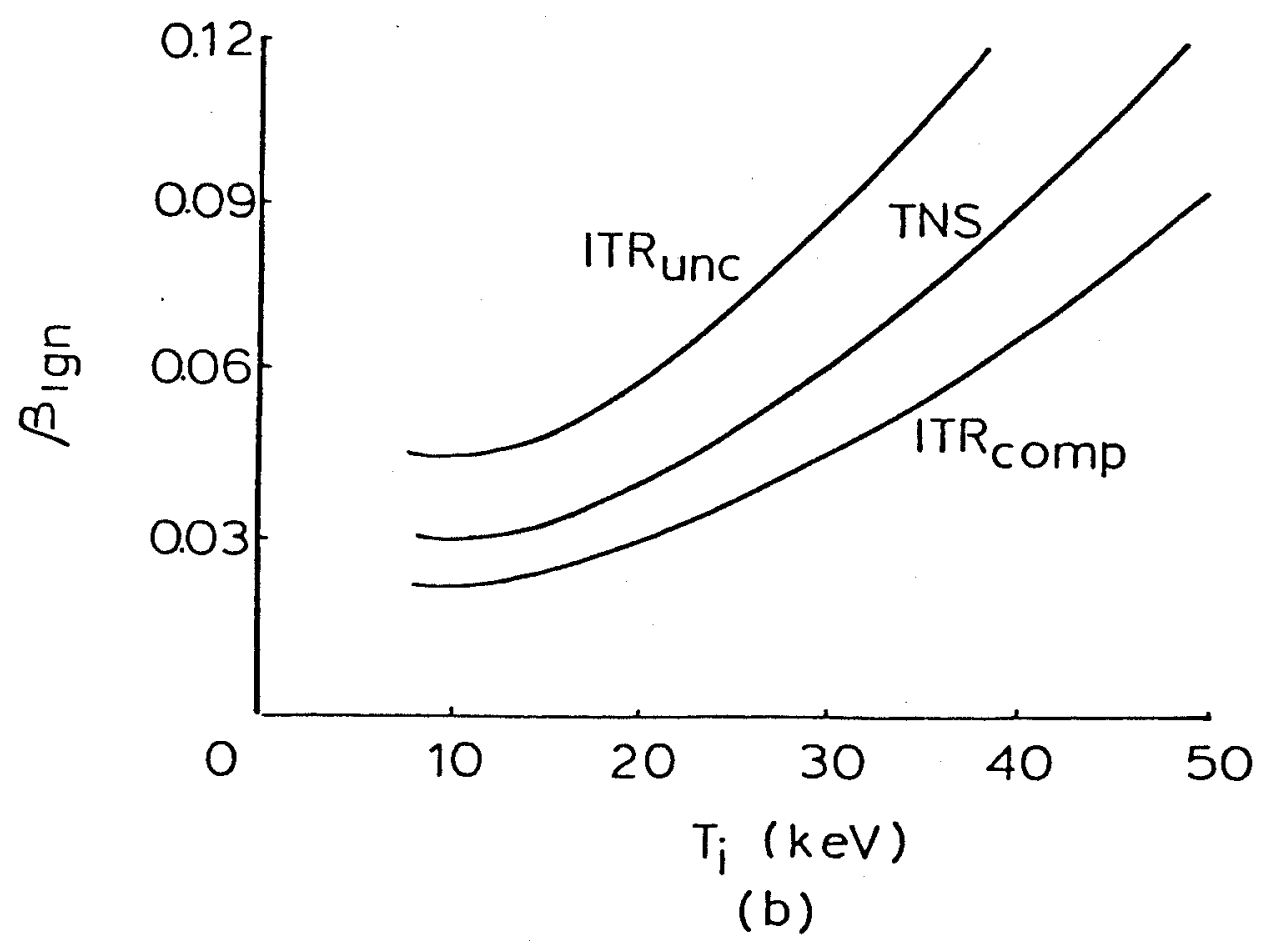

Figure 7 
Page 24

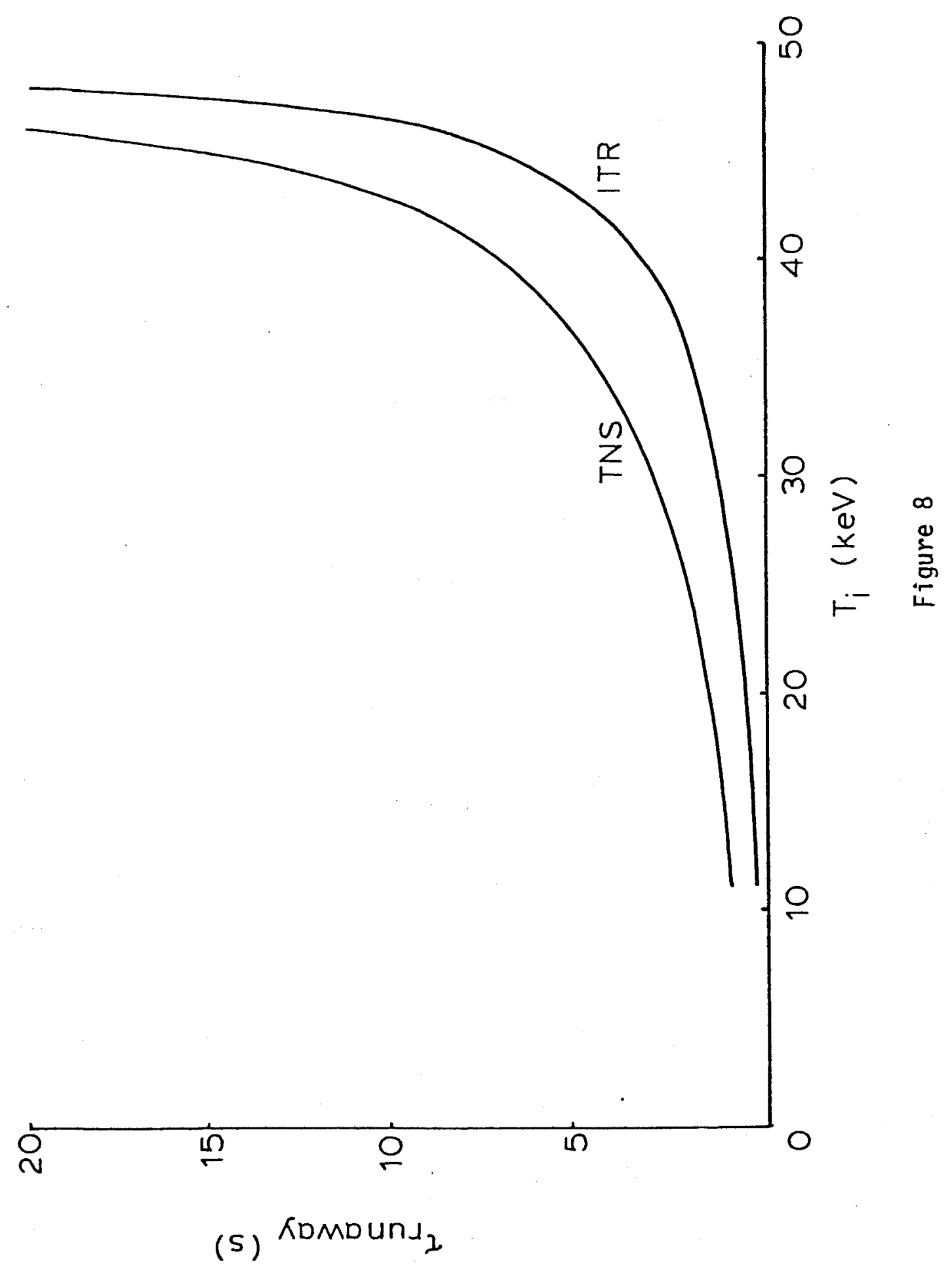


Page 25

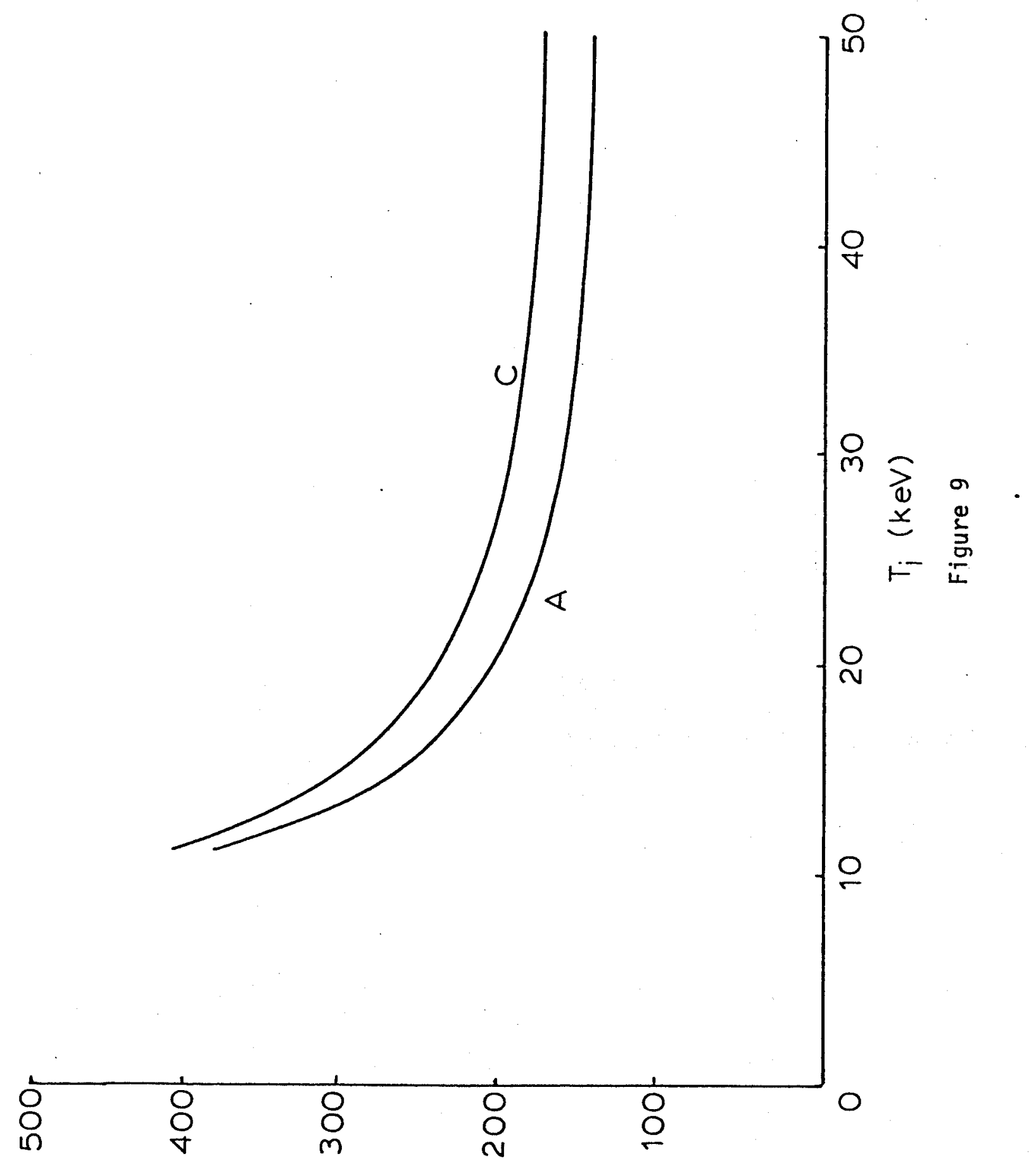

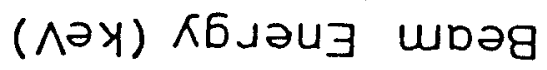

